\title{
Possibility to deduce the emission time sequence of neutrons and protons from the neutron-proton correlation function?
}

\author{
R. Ghetti ${ }^{a, 1}$, J. Helgesson ${ }^{b}$, N. Colonna ${ }^{c}$, B. Jakobsson ${ }^{a}$, A. Anzalone ${ }^{d}$, V. Bellini ${ }^{d, e}$, L. Carlèn ${ }^{a}$, S. Cavallaro ${ }^{d, e}$, \\ L. Celano ${ }^{c}$, E. De Filippo ${ }^{e, f}$, G. D'Erasmo ${ }^{c}$, D. Di Santo ${ }^{c}$, E.M. Fiore ${ }^{c}$, A. Fokin ${ }^{a}$, M. Geraci ${ }^{f}$, F. Giustolisi ${ }^{e}, f$,

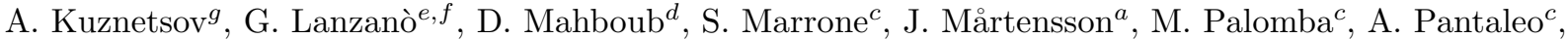

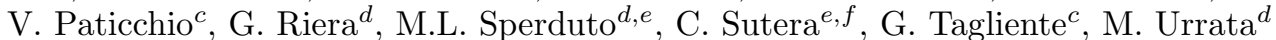 \\ ${ }^{a}$ Department of Physics, Lund University, Box 118, SE-221 00 Lund, Sweden \\ ${ }^{b}$ Malmö University, School of Technology and Society, SE-205 06 Malmö, Sweden \\ ${ }^{c}$ INFN and Dipartimento di Fisica, Via Amendola 173, I-70126 Bari, Italy \\ ${ }^{d}$ Laboratori Nazionali del Sud (INFN), Via S. Sofia 44, I-95123 Catania, Italy \\ ${ }^{e}$ Dipartimento di Fisica, Università di Catania, Corso Italia 57, I-95129 Catania, Italy \\ ${ }^{f}$ INFN, Sezione di Catania, Corso Italia 57, I-95129 Catania, Italy \\ ${ }^{g}$ Khlopin Radium Institut, Shvernik Avenue 28, 194021 St. Petersburg, Russia
}

(November 3, 2018)

Experimental information has been derived from the neutron-proton correlation function in order to deduce the time sequence of neutrons and protons emitted at $45^{\circ}$ in the $\mathrm{E} / \mathrm{A}=45 \mathrm{MeV}^{58} \mathrm{Ni}+{ }^{27} \mathrm{Al}$ reaction.

PACS number(s): 25.70.Pq, 29.30.Hs

${ }^{1}$ Corresponding Author: Roberta Ghetti, Department of Physics, Lund University, Box 118, SE-221 00 Lund, Sweden Tel. +46-46-2227388, Fax +46-46-2224015

Two-nucleon correlation functions are normally utilized to extract information on the size of the emitting source and on the time duration of the emission [1]. Furthermore, when correlations of non-identical particles are considered, additional model-independent information on the emission chronology of the particles can be obtained.

A technique to probe the emission sequence and time delay of ejectiles in nuclear reactions was first suggested for charged particle pairs [2], based on the idea that mutual Coulomb repulsion would be experienced by pairs of charged particles emitted with a short time delay. Comparison of the velocity difference spectra with trajectory calculations would thus give a measure of the average particle emission sequence [3]. The technique was extended to any kind of interacting, non-identical particle pairs in the theoretical study of Ref. [4]. There it was demonstrated that the sensitivity of the correlation function to the asymmetry of the distribution of the relative spacetime coordinates of the particle emission points can be used to determine the differences in the mean emission times. This effect has been discussed for different particle pairs, for instance $p d, n p$ [4], $\pi p$ [5], $K^{+} K^{-}$[6]. The technique of Ref. [4 has been applied in recent experimental studies where the time sequence for $p$ and $d$ emitted in the $\mathrm{E} / \mathrm{A}=50 \mathrm{MeV} \mathrm{Xe}+\mathrm{Sn}$ reaction [7] and for $p, d, t,{ }^{3} \mathrm{He}$ and $\alpha$ particles emitted in central $\mathrm{E} / \mathrm{A}=400$ $\mathrm{MeV} \mathrm{Ru}+\mathrm{Zr}$ collisions [8] has been deduced. Typical time delays of a few $\mathrm{fm} / \mathrm{c}$ have been found in [8].

In this paper we report on the first experimental evidence that the emission chronology of neutrons and pro- tons can be deduced from the neutron-proton $(n p)$ correlation function. These data come from the $\mathrm{E} / \mathrm{A}=45$ $\mathrm{MeV}^{58} \mathrm{Ni}+{ }^{27} \mathrm{Al}$ reaction 9 11]. After a short discussion about the possibility to obtain chronology information, clarified with the help of theoretical calculations of the effects, indications on the emission sequence are derived from the experimental ungated $n p$ correlation function as well as from the correlation function gated on the parallel velocity and on the total momentum of the nucleon pair.

The possibility to access information from the $n p$ correlation function on the particle emission time sequence is connected to the different strength of the nuclear final state interaction experienced by different pairs. Namely, from a merely classical viewpoint, if there are pairs for which the average distance between the two particles (when the two particles start to interact) is smaller, these pairs experience a stronger interaction and exhibit an enhanced correlation (or anticorrelation) strength, as compared to those pairs for which the average particle distance is larger. Now, if there is an average time difference in the emission times between the two particles, there will also be a difference in the average distance for the two "classes" $E_{n}>E_{p}$ and $E_{n}<E_{p}$.

In order to study the $n-p$ emission chronology, one can calculate the $n p$ correlation function with different source models and parameter sets. Due to the strong final state interaction between $n$ and $p$, the $n p$ correlation function shows a strong correlation for small values of the relative momentum $q$ and, for some parameter sets, a weak anticorrelation for intermediate values of $q$. This is due to the fact that the correlation in the singlet channel (antiparallel spins of $n p$ ) is positive while it is negative in the triplet channel (parallel spins of $n p$ ) even if the interaction in both channels is attractive. The counterintuitive negative correlation in the triplet channel is due to the deuteron formation. Because of this process, the sample of $n p$ pairs is depleted and one effectively observes the negative correlation [12].

If a time delay in the emission of the two particles is introduced, the interaction is enhanced for those pairs for 
which the average distance is smaller. This can be easily seen if one compares the correlation function $C_{n}(q)$, gated on pairs $E_{n}>E_{p}$, with the correlation function $C_{p}(q)$, gated on pairs $E_{n}<E_{p}$. If the proton is emitted earlier (later) than the neutron, the ratio $C_{n} / C_{p}$ will show a peak (dip) in the region of $q$ where there is a correlation, a dip (peak) where there is an anticorrelation, and will approach unity both for $q \rightarrow 0$ (since the energy difference of the two emitted particles is negligible) and $q \rightarrow \infty$ (since modifications of the two-particle phase space density arising from final state interactions are negligible). The exact location of the peak and dip in the ratio depends on the source model and on the parameter set (as does of course the correlation function itself).

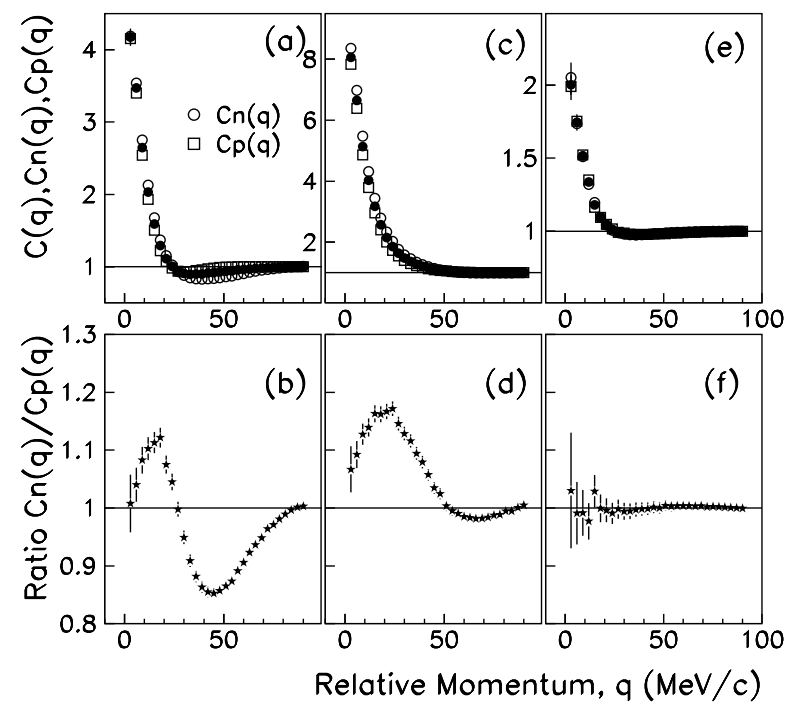

FIG. 1. Calculations performed with the source model of Ref. [14]. Panels (a)(b): $n p$ correlation functions $C(q)$ (solid dots), $C_{n}(q), C_{p}(q)$ and the ratio $C_{n} / C_{p}$, from a source emitting $p$ and $n$ with emission time width of $\Delta t=40 \mathrm{fm} / \mathrm{c}$ and with the neutron emission time delayed $50 \mathrm{fm} / \mathrm{c}$. Panels $(c)(d)$ : the same as in panels $(a)(b)$, obtained with emission time width of $\Delta t=80 \mathrm{fm} / \mathrm{c}$. Panels (e)(f): the same as in panels $(\mathrm{a})(\mathrm{b})$, from a "two-source model" calculation [10], with exponential lifetime of $\tau_{p} \approx 400 \pm 200 \mathrm{fm} / \mathrm{c}$ for protons, $\tau_{n} \approx 600 \pm 200 \mathrm{fm} / \mathrm{c}$ for neutrons $(\approx 30 \%$ of $p$ and $\approx 13 \%$ of $n$ come from pre-equilibrium).

This effect has been calculated in Ref. [4] for different delays in the emission of neutrons and protons. The calculation for the $n p$ system assuming that protons are on average emitted $100 \mathrm{fm} / \mathrm{c}$ earlier than neutrons (right panels in Fig. 2 of Ref. [4), shows $C_{n}(q)<1$ down to $q \approx$ $15 \mathrm{MeV} / \mathrm{c}$ and a corresponding $\operatorname{dip}$ in the ratio $C_{n} / C_{p}$. The expected peak at small $q$, where the correlation function shows a strong correlation, is washed out in these calculations, since the statistics is very low and the parameter set generates a correlation only in the very low $q$-region $(<15 \mathrm{MeV} / \mathrm{c})$, where the ratio $C_{n} / C_{p}$ has to approach unity [13].

To illustrate this point further, we present in Fig. 11 three model calculations performed with the source model of Ref. 14] coupled to the Koonin-Pratt formalism [15] to include final state interactions. The first calculation represents the $n p$ correlation function obtained from the source model of Ref. [14] with time width parameter $\Delta t=40 \mathrm{fm} / \mathrm{c}$ and with the neutron emission time delayed $50 \mathrm{fm} / \mathrm{c}$ with respect to the proton emission time. Fig. 1 a shows the calculated $C(q)$ (solid dots), $C_{n}(q)$ (open circles) and $C_{p}(q)$ (open squares). The ratio $C_{n} / C_{p}$ is shown in Fig. 1 b. $C(q)$ presents a weak anticorrelation in the intermediate $q$-region between 30 and $50 \mathrm{MeV} / \mathrm{c}$ and a strong correlation at $q<20 \mathrm{MeV} / \mathrm{c}$. The correlation function $C_{n}(q)$ gated on pairs $E_{n}>E_{p}$ demonstrates an enhanced interaction, since the $n-p$ average distance when the particles start to interact is shorter for these pairs. The ratio $C_{n} / C_{p}$ is above unity where $C(q)$ has a correlation and below unity where $C(q)$ has an anticorrelation Figs. 11c, 11d illustrate the results of a similar calculation with larger width of the time distribution $\Delta t=80 \mathrm{fm} / \mathrm{c}$. One can see that the anticorrelation in $C(q)$ is weaker and shifted to larger values of $q$. The ratio $C_{n} / C_{p}$ is changed accordingly. Finally Figs. 11e, $1 \mathrm{f}$ present the results of the "two-source" calculation of Ref. [10], where it is assumed that $\approx 30 \%$ of the protons and $\approx 13 \%$ of the neutrons come from pre-equilibrium emission. The parameter set used for this calculation yields emission time distributions with exponential lifetime values of $\tau_{p} \approx 400 \pm 200 \mathrm{fm} / \mathrm{c}$ for protons and $\tau_{n} \approx 600 \pm 200$ $\mathrm{fm} / \mathrm{c}$ for neutrons. The ratio $C_{n} / C_{q}$ remains close to unity for all values of $q$.

The experimental $n p$ correlation function from the $\mathrm{E} / \mathrm{A}=45 \mathrm{MeV}{ }^{58} \mathrm{Ni}+{ }^{27} \mathrm{Al}$ reaction, obtained with a proton detection array placed at $45^{\circ}$ and a neutron setup of liquid scintillators placed at $25^{\circ}, 45^{\circ}$ and $90^{\circ}$, was presented in Ref. [10] and is shown by the solid dots in Figs. 2a, 2 b. Kinetic energy thresholds of 2-50 MeV for neutrons and 6-50 MeV for protons are applied to the data. The normalization is defined in the region $q=80-120$ $\mathrm{MeV} /$ c. $C(q)$ shows a clear correlation for $q<20 \mathrm{MeV} / \mathrm{c}$ and a very small anticorrelation for $20<q<70 \mathrm{MeV} / \mathrm{c}$.

In order to study the $n-p$ emission chronology, the correlation function $C_{n}(q)$, gated on pairs $E_{n}>E_{p}$, and $C_{p}(q)$, gated on pairs $E_{n}<E_{p}$, are presented in Fig. 2 together with the ratio $C_{n} / C_{p} . E_{n}$ and $E_{p}$ are calculated in the reference frame of a source moving with a velocity of $0.16 \mathrm{c}$ [1]. A single normalization constant, calculated from the ungated correlation function, is utilized for both energy-gated correlation functions $C_{n}(q)$ and $C_{p}(q)$.

The data show a small experimental signal. $C_{n}(q)$ is slightly enhanced with respect to $C(q)$ for all values of $q<60 \mathrm{MeV} / \mathrm{c}$ (Fig. 2a). Correspondingly $C_{p}(q)$ is suppressed in the same $q$-region (Fig. 2 $\mathrm{b}$ ). The ratio $C_{n} / C_{p}$ is above unity for $q<70 \mathrm{MeV} / \mathrm{c}$ (Fig. 2 2c). The signal has proved to be rather stable. When testing it against the accuracy of the energy calibrations, it turns out that 
a statistical uncertainty of up to $20-30 \%$ does not have any appreciable effects on the $C_{n} / C_{q}$ ratio. On the other hand, a systematic error of the same size might significantly alter the final results, but we believe that the accuracy of our energy calibrations is within $5-10 \%$ [10].

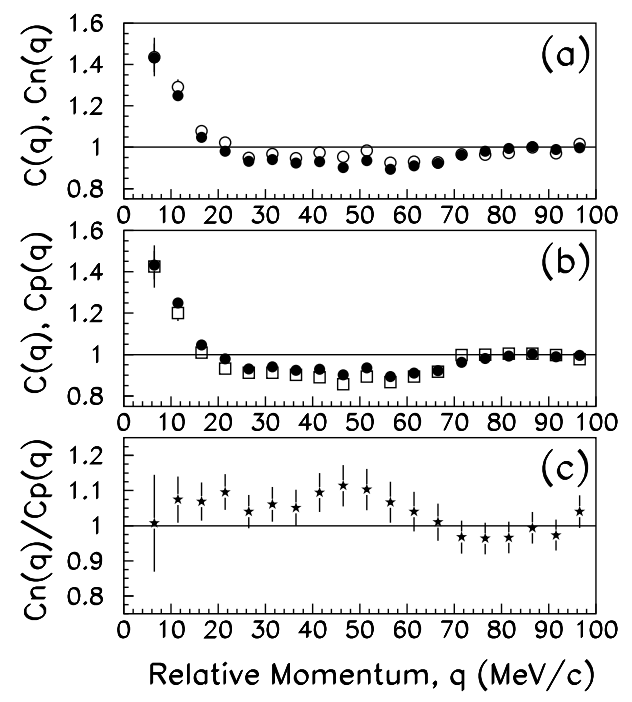

FIG. 2. Experimental ungated $n p$ correlation function $C(q)$, from the $\mathrm{E} / \mathrm{A}=45 \mathrm{MeV}{ }^{58} \mathrm{Ni}+{ }^{27} \mathrm{Al}$ reaction, (solid dots in panels (a)(b)) compared to: Panel (a), open circles: $C_{n}(q)$, constructed from pairs of type $E_{n}>E_{p}$. Panel (b), open squares: $C_{p}(q)$, constructed from pairs of type $E_{n}<E_{p}$. The ratio $C_{n} / C_{p}$ is shown in panel (c).

As discussed above, the enhancement of $C_{n}(q)$ where $C(q)$ has a correlation $(q<20 \mathrm{MeV} / \mathrm{c})$ might indicate that for those pairs that contribute to the region $q<20$ $\mathrm{MeV} / \mathrm{c}$ protons are on average emitted earlier than neutrons. The enhancement of $C_{n}(q)$ in the region $20<q<$ $60 \mathrm{MeV} / \mathrm{c}$ might instead indicate the opposite emission time sequence as $C(q)$ has an anticorrelation there. However, one should keep in mind that the inclusive correlation function reflects a convolution of different sources and origins of emission thus it is not reasonable to expect that the information from the inclusive data should provide us with a well defined and unique $n p$ emission time sequence.

In order to disentangle the different effects and minimize such averaging, various kinds of gates can be applied to the inclusive data. We shall now present correlation functions with additional gates and we shall see that indeed, despite the poor statistics, the gated correlation functions yield a ratio $C_{n} / C_{p}$ in closer accordance with the qualitative appearance of Fig. 1 .

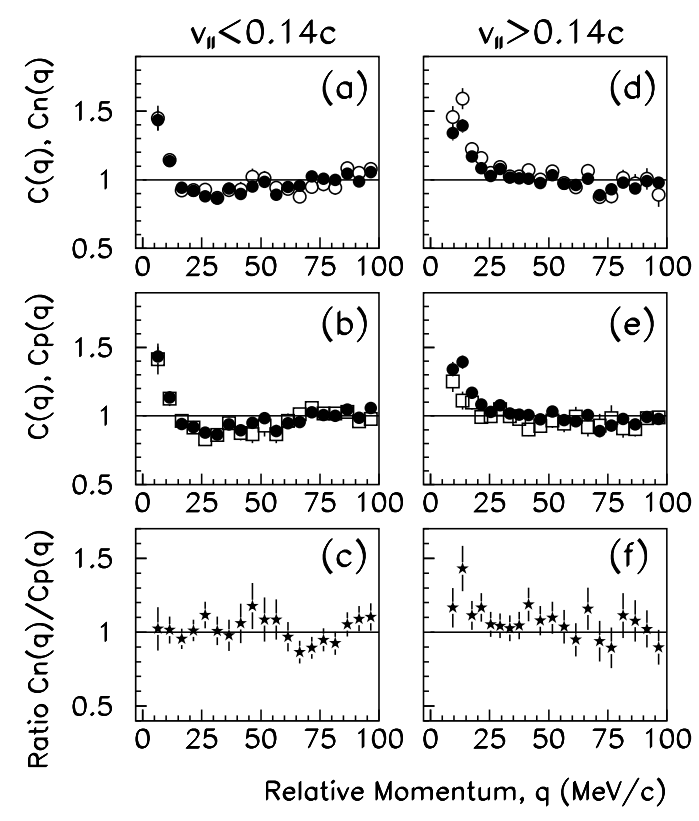

FIG. 3. The same as in Fig. 2, for low- $v_{\|}(<0.14 \mathrm{c}) n p$ pairs (panels $(\mathrm{a})(\mathrm{b})(\mathrm{c}))$ and for high- $v_{\|}(>0.14 \mathrm{c}) n p$ pairs (panels $(d)(e)(f))$.

Fig. 3 illustrates how the results of Fig. 2 are modified when cuts on the parallel velocity of the particles are applied. The effects of these cuts, that are defined on the basis of BUU calculations [16] and of the available statistics, are to enhance (suppress) the quasi-projectile source by selecting nucleons with large (small) values of the parallel velocity. Figs. 3a, 3b, 3c correspond to a low$v_{\|}$gate and Figs. $3 \mathrm{~d}$, 3e, 3f to a high- $v_{\|}$gate. One can notice that the behavior of the correlation functions and of $C_{n} / C_{p}$ ratio is quite different for the two different event selections. "Projectile-like" events (i.e. high- $v_{\|}$selection) exhibit an enhanced correlation function strength and an enhanced $C_{n} / C_{p}$ ratio in correspondence to the correlation $(q<25 \mathrm{MeV} / \mathrm{c})$. "Target-like" events (i.e. low- $v_{\|}$ selection) exhibit a slight suppression in the correlation function strength $(q<20 \mathrm{MeV} / \mathrm{c})$, a weak anticorrelation for $q \approx 20-50 \mathrm{MeV} / \mathrm{c}$ and a $C_{n} / C_{p}$ ratio quite close to unity in the correlation region.

Gating on the momentum (or energy) of the particle pair in the source system is particularly useful to investigate the time-scale of the emission and to study the interplay between dynamical and statistical effects in particle emission. The $n p$ correlation function gated on high- $P_{t o t}$ pairs has already been presented in Ref. [10]. An enhancement in the correlation function strength was observed indicating a smaller space-time extent of the pre-equilibrium source of emission [10]. Fig. 囵 presents total-momentum-gated correlation functions. A low- $P_{t o t}$

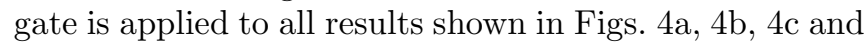

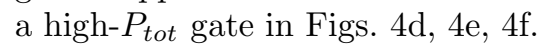

The effects of the gates on the correlation functions 
and on the $C_{n} / C_{p}$ ratio are similar to those observed in Fig. 3. High-total-momentum selected events exhibit an enhanced correlation function strength and an enhanced $C_{n} / C_{p}$ ratio in correspondence to the correlation $(q<25$ $\mathrm{MeV} / \mathrm{c}$ ). Low-total-momentum selected events exhibit a slight suppression in the correlation function strength $(q<20 \mathrm{MeV} / \mathrm{c})$, a weak anticorrelation in the intermediate $q$-region and a $C_{n} / C_{p}$ ratio that does not appreciably deviate from unity at any values of $q$.

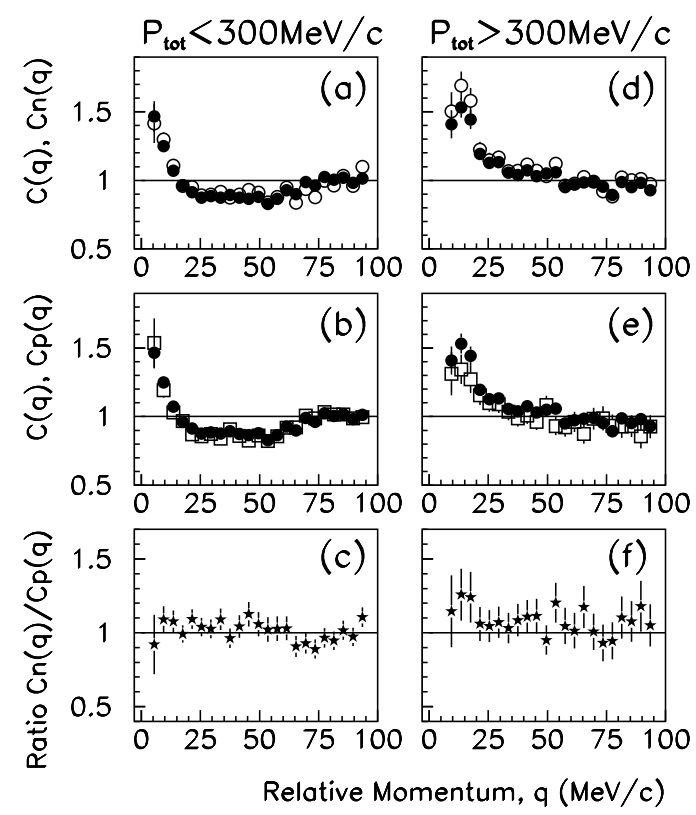

FIG. 4. The same as in Fig. 2, for low- $P_{t o t}(<300 \mathrm{MeV} / \mathrm{c})$ $n p$ pairs (panels $(\mathrm{a})(\mathrm{b})(\mathrm{c}))$ and for high- $P_{\text {tot }}(>300 \mathrm{MeV} / \mathrm{c})$ $n p$ pairs (panels $(\mathrm{d})(\mathrm{e})(\mathrm{f})) . P_{\text {tot }}$ is calculated in the reference frame of a source moving with $v_{\text {source }}=0.16 \mathrm{c}$.

The enhancement of the $C_{n} / C_{p}$ ratio in the low- $q$ region, observed in Figs. $3 \mathrm{f}$ and $4 \mathrm{f}$, could be interpreted as an indication that for those $n p$ pairs, protons are on the average emitted earlier than neutrons.

The result of a shorter average emission time for protons seems to corroborate our previous findings from $n n$ and $p p$ data compared to a two-component statistical model 10]. However, the calculation of the $C_{n} / C_{p}$ ratio with the "two-source model" parameters used in Ref. [10 yields a negligible effect (shown in Fig. 1f) as compared to the experimental data (Figs. $3 f$ and $4 \mathrm{f}$ ). While this might indicate that the initial time distribution of the "two-source model" calculation is too broad and/or wrongly shaped, one should also keep in mind that there could be alternative explanations. The model of [10] neglects any residual interaction between the emitted particles and the reaction zone. In particular, the Coulomb interaction between the proton and the emitting source - which could increase the average distance between the two particles if the proton is emitted first - has not been taken into account 10], although, according to Ref. [4, the influence of the Coulomb field of the residual nucleus on the proton is expected to be minimal. Alternative explanations might be found in reaction mechanisms that are neglected in our calculations, such as delayed feeding from the decay of excited primary fragments. It thus appears as if a refined theoretical treatment would be welcome to confirm or not the interpretation presented here.

In conclusion, the experimental results from differently gated correlation functions are in qualitative agreement with a classical argumentation and calculations using the Koonin-Pratt formalism [15], and support the interpretation that in high-parallel-velocity and high-totalmomentum selected events, which enhance projectile-like and/or intermediate velocity sources, the proton is on average emitted earlier than the neutron. Although alternative explanations of the enhancement in the $C_{n} / C_{p}$ ratio cannot be ruled out at this time, the experimental evidence of such an enhancement, presented here for the first time, is by itself intriguing and deserves further experimental and theoretical investigation.

The authors would like to thank Prof. S. Mrowczynski for valuable discussion. The support from the Swedish Natural Science Research Council is appreciated.

[1] For a review, see e.g. D. Ardouin, Int. Journ. Mod. Phys. E 6 (1997) 391.

[2] C.J. Gelderloos and J.M. Alexander, Nucl. Inst. Meth. A 349 (1994) 618.

[3] C.J. Gelderloos, et al., Phys. Rev. Lett. 75 (1995) 3082; C.J. Gelderloos, et al., Phys. Rev. C 52 (1995) R2834.

[4] R. Lednicky, V.L. Lyuboshitz, B. Erazmus, D. Nouais, Phys. Lett. B 373 (1996)30.

[5] S. Voloshin, R. Lednicky, S. Panitkin and N. Xu, Phys. Rev. Lett. 79 (1997) 4766.

[6] D. Ardouin, et al. Phys. Lett. B 446 (1999) 191.

[7] D. Gourio, et al., Eur. Phys. J. A 7 (2000) 245.

[8] R. Kotte and H.W. Barz, Eur. Phys. J. A 6 (1999) 185.

[9] R. Ghetti, et al., Nucl. Phys. A660 (1999) 20.

[10] R. Ghetti, et al., Nucl. Phys. A674 (2000) 277.

[11] R. Ghetti, et al., Phys. Rev. C 62 (2000) 037603.

[12] S. Mrowczynski, Phys. Lett. B 277 (1992) 43.

[13] R. Lednicky, private communication (Sept. 2000).

[14] J. Helgesson, T. Csörgo, M. Asakawa and B. Lörstad, Phys. Rev. C 56 (1997) 2626.

[15] S.E. Koonin, Phys. Lett. B 70 (1977) 43; S. Pratt, M.B. Tsang, Phys. Rev. C 36 (1987) 2390.

[16] R. Ghetti, et al., Phys. Rev. C 64 (2001) 017602. 